\title{
MAINE DE BIRAN E HENRI BERGSON. LEITURAS CONTEMPORÂNEAS DE UMA TESE PARTILHADA
}

\section{LUÍS ANTÓNIO UMBELINO1}

RESUMO: O presente trabalho pretende posicionar-se no horizonte da longa e interessante história das aproximações tentadas entre o pensamento de Maine de Biran e de H. Bergson. Mais especificamente pretende-se identificar pelo menos um dos pontos de convergência que, sem ignorar as respetivas distâncias, justificaram na contemporaneidade filosófica a recuperação da aproximação entre Biran e Bergson. Neste sentido, gostaríamos de argumentar que tal abeiramento (que comprova não terem sido nem fortuitas, nem ligeiras as referências elogiosas de Bergson a Biran) se patenteia - como já sugeriu P. Montebello - em redor de um entendimento comum dos perigos e equívocos funestos do denominado paralelismo psicofisiológico. Será por aqui que avançaremos neste estudo.

PALAVRAS-CHAVE: Maine de Biran; Henri Bergson; Paralelismo psicofisiológico.

ABSTRACT: This paper intends to position itself on the horizon of the long and interesting history of the attempted approximations between the thoughts of Maine de Biran and $\mathrm{H}$. Bergson. More specifically, it is intended to identify at least one of the convergence points that, without ignoring the respective distances, justified in the philosophical contemporaneity the recovery of the approximation between Biran and Bergson. In this sense, we would like to argue that such an approach (which proves to be neither fortuitous, nor occasional Bergson's praiseworthy references to Biran) shows - as P. Montebello has already suggested - around a common understanding of the dangers and misconceptions of the so called psychophysiological parallelism. This is where we will advance in this study.

KEYWORDS: Maine de Biran; Henri Bergson; Psychophysiological parallelism.

\section{Aproximações e diferenças.}

As tentativas de aproximação entre o pensamento de Maine de Biran e de H. Bergson têm uma história longa e interessante, porventura legitimada pelas menções inequivocamente elogiosas de Bergson à filosofia biraniana em textos como Rapport sur le prix Bordin' ${ }^{2}$, de 1905, ou La philosophie française, trabalho de1915. Neste último, por exemplo, Bergson afirmava de modo sugestivo o seguinte:

\footnotetext{
${ }^{1}$ Professor do Departamento de Filosofia, Comunicação e Informação da Faculdade de Letras da Universidade de Coimbra. Membro da Unidade I\&D CECH. Doutor em Filosofia pela Faculdade de Letras da Universidade de Coimbra. E-mail: lumbelino@fl.uc.pt.

${ }^{2}$ Henri Bergson, « Rapports sur le prix Bordin. Concours ayant pour sujet Maine de Biran et sa place dans la philosophie moderne », in ID, Mélanges, Édition du Centenaire (Paris : PUF, 1972), 662-671.
} 
desde o começo do século, a França teve um grande metafísico, o maior que produziu desde Descartes e Malebranche. Pouco notada no momento em que surgiu, a doutrina de Maine de Biran exerceu uma influência crescente: pode-se perguntar se a via que este filosofo abriu não é aquela pela qual a metafísica deverá caminhar definitivamente. ${ }^{3}$

O leitor competente de Bergson sabe bem a que ponto cada uma das palavras vertidas da pena do filósofo é sopesada de forma minuciosa, a que ponto cada frase apenas se termina depois de apurado trabalho de meditação e de polimento da forma, a que ponto é assumido o escrúpulo de nada dizer a mais e de nada dizer a menos do aquilo que se quer efetivamente afirmar. Uma referência assim tão clara e franca a Biran não terá sido, pois, apenas circunstancial: indicia-se uma ligação, uma receção marcante, algum tipo de acordo de fundo.

Será com esta convicção, julgamos, que vários comentadores reputados, como sejam, por exemplo, Jean Laporte, Gabriel Madinier ${ }^{4}$;Marguerite Thibaud ${ }^{5}$, ouGeorges Le Roy ${ }^{6}$, entenderão ser importante proceder à ponderação comparativa dos dois projetos filosóficos. Reveste-se de igual importância o momento em que, sobre um tal estudo comparativo, se reconhecem mais significativas as distâncias do que as adjacências. Ilustra essa circunstância, nomeadamente, o modo como H. Gouhier, sem ignorar os já referidos contributos e propostas de leitura cotejada, defende - em textos como "Maine de Biran et Bergson"7 - que apenas o reconhecimento do significado filosófico das dissemelhanças de fundo entre os dois projetos permitirá concretizar um efetivo e profícuo estudo comparativo. Na linha desta mesma história, não deixa de ser interessante notar ainda o modo como certa contemporaneidade filosófica recuperará, sobre novas bases e em face de problemas resistentes,o significado filosófico de um abeiramento necessário, como se apenas este permitisse medir a atualidade dos dois projetos filosóficos dissemelhantes. Ilustrará seguramente esta possibilidade, por exemplo, o pequeno texto que Deleuze, poucos meses antes de suicidar, dedica uma última vez ao tema da imanência - subtilmente esclarecida como sendo "uma vida" qual convoca as referências de Biran e de Bergson, porventura sugerindo que os une a convicção comum de que a vida consciente se desvenda enquanto expressão da nossa existência fundamentalmente corpórea e viva. Poderia igualmente elencar-se aqui o modo

\footnotetext{
${ }^{3}$ Henri Bergson "La Philosophie Française”, in Revue de Paris, mai (1915), p. 247, cit. in H. Gouhier, « Maine de Biran et Bergson », in Les études bergsoniennes, vol. 1 (1948), (Paris : Albin Michel), 145.

${ }^{4}$ Gabriel Madinier, Conscience et mouvement. Étude sur la philosophie française de Condillac à Bergson, (Paris : Alcan, 1938).

${ }^{5}$ Marguerite Thibaud, L'effort chez Maine de Biran et Bergson, (Grenoble : Imprimerie Allier \& Fils, 1939).

${ }^{6}$ George le Roy, L'expérience de l'effort et de la grâce chez Maine de Biran, (Paris : Boivin \& Cie., 1937).

${ }^{7}$ Henri Gouhier «Maine de Biran et Bergson», in Les études bergsoniennes, 1 (1948), 131-173.

${ }^{8}$ Gilles Deleuze, «L'Immanence : une vie », in Philosophie, 47(1995), 3-7.
} 
como G. Simondon, em L'individu et sa genèse phisico-biologique, sugere uma linha de argumentação que passa tanto por Biran como por Bergson, algo notado e explorado de modo original por P. Montebello que, por seu turno, se proporá, em Nature et Subjectivité, colocar "Simondon sobre uma linha que passa de Biran a Bergson e Deleuze" ${ }^{9}$. A esta rede densa de referências, poderia ainda acrescentar-se a menção a momentos precisos do projeto original de R. Barbaras no qual o filósofo francês assume situar-se "entre Husserl e Bergson", num espaço de reflexão onde, de algum modo, se encontrará entre Patočka e a influência biraniana assumida por este último ${ }^{10}$.

Deixaremos para trabalhos futuros o inventário completo desta malha cerrada de alusões. Nesta ocasião, o nosso objetivo será mais modesto: identificar pelo menos um dos pontos de convergência que, sem ignorar as respetivas distâncias, justificaram na contemporaneidade filosófica a recuperação da aproximação entre Biran e Bergson. Neste sentido, gostaríamos de argumentar que tal abeiramento (que comprova não terem sido nem fortuitas, nem ligeiras as referências elogiosas de Bergson a Biran) se patenteia - como já sugeriu P. Montebello -em redor de um entendimento comum dos perigos e equívocos funestos do denominado paralelismo psicofisiológico. Será por aqui que avançaremos neste estudo.

\section{A questão do paralelismo psicofisiológico.}

Em "L’âme et le corps"11 e em "Le cerveau et la pensée, une illusion philosophique"12 Bergson regressa explicitamente (ainda no horizonte das perspetivas e questões abertas pelos primeiros capítulos de Matière et mémoire) ao problema filosófico maior das relações entre corpo e espírito. Fá-lo com o intuito de enfrentar criticamente a resposta que a filosofia moderna aportou a tal problema: uma resposta assente na tese de um "paralelismo entre alma e corpo". Tal paralelismo corresponde à ideia funesta "de uma equivalência entre um estado psíquico e um estado cerebral correspondente"13, ideia que, argumentará Bergson, se funda na

\footnotetext{
${ }^{9}$ Pierre Montebello, Nature et Subjectivité, (Grenoble : Millon, 2007), p. 93.

${ }^{10} \mathrm{Cf}$., por exemplo, Renaud Barbaras, Introduction à une philosophie de la vie (Paris ; Vrin, 2008).

${ }^{11}$ Henri Bergson, "L'âme et le corps", in ID L'énergie spirituelle (édition critique sous la direction de F. Worms), (Paris : PUF, Quadrige, 2009), 29-60

${ }^{12}$ Henri Bergson, "Le cerveau et la pensée, une illusion philosophique",in ID L'énergie spirituelle (édition critique sous la direction de F. Worms), (Paris : PUF, Quadrige, 2009), 191-210

${ }^{13}$ Bergson, L'énergie spirituelle, 191.
} 
convicção acrítica segundo a qual seria possível "ler num cérebro tudo o que se passa na consciência correspondente."14

A atualidade de Bergson nesta matéria é a de ter reconhecido que um tal ensejo de legibilidade integral do pensamento é uma mera hipótese que se aceita sem recurso à observação, demonstração ou evidência empírica. O mesmo é dizer que tal hipótese não nasceu da observação de dados científicos, algo que se pode demostrar historicamente pelo facto de ser mais antiga do que as primeiras conquistas consequentes da fisiologia e anatomia do cérebro; a sua origem, de acordo com Bergson, não será, portanto, o conjunto das conquistas científicas que pretendem inferir do funcionamento cerebral o fenómeno do pensamento, antes devendo ser procurada em "princípios gerais de uma metafísica que se concebeu, pelo menos em grande medida, para dar corpo às esperanças da física moderna" ${ }^{15} \mathrm{e}$ da fisiologia. É a suposição metafísica - e não a certeza científica - de uma traduzibilidade ou legibilidade integral do psíquico no físico que, portanto, operará acriticamente sob a marcha de tais estudos no momento em que procuram explicar a "relação" entre o pensamento e o cérebro. E quando uma tal conjetura metafísica é autorizada a orientar a investigação, a explicação da relação rapidamente se orientará pela ideia infundada segunda a qual "estando dado um estado cerebral, segue-se um estado psicológico determinado"16, estando dada uma estrita equivalência entre estados cerebrais e estados de consciência, nada obstará, no limite, a que se aceite a ideia segundo a qual o pensamento não é senão "uma função do cérebro"17.

O postulado de um tal paralelismo encerra, no entanto, de acordo com Bergson, "uma contradição fundamental" ${ }^{\prime 18}$ e uma confusão básica de pontos de vista ${ }^{19}$ que importa denunciar por configurarem um "artifício dialético": a tese do paralelismo psicofisiológico promove a passagem sub-reptícia entre inconciliáveis "sistemas de notação" ${ }^{20}$ (idealista e realista), ao dissimular a contradição insolúvel que existe entre as conceções de matéria e de espirito com as quais forçosamente se opera ao usar tais sistemas de notação ${ }^{21}$; deste modo, o recurso a explicações que se fundam na tese do paralelismo não serve realmente para solucionar o problema da passagem entre pensamento e cérebro, permanecendo uma estratégia ou

\footnotetext{
${ }^{14}$ Bergson, L'énergie spirituelle, 36.

${ }^{15}$ Bergson, L'énergie spirituelle, 39; 192: “Mais l'affirmation dogmatique du parallélisme psychophysiologique est toute autre chose. Ce n'est plus une règle scientifique, c'est une hypothèse métaphysique. »

${ }^{16}$ Bergson, L'énergie spirituelle, 191.

${ }^{17}$ Bergson, L'énergie spirituelle, 38.

${ }^{18}$ Bergson, L'énergie spirituelle, 193.

${ }^{19}$ Bergson, L'énergie spirituelle, 198 : “Mais la vérité est qu'on passe inconsciemment du point de vue idéaliste à un point de vue pseudo-réaliste. »

${ }^{20}$ Bergson, L'énergie spirituelle, 195 ss.

${ }^{21}$ Bergson, L'énergie spirituelle, 195-196
} 
"artifício dialético" que apenas ilude o carácter problemático de tal passagem. Será sobre este artifício que se configurará toda uma mitologia propriamente moderna em relação a estas matérias: uma mitologia onde a personagem principal será

uma inteligência sobre-humana, que assistiria à dança dos átomos de que o cérebro é feito e que teria a chave da psicofisiologia, [e assim] poderia ler, no cérebro que trabalha, tudo o que se passa na consciência correspondente. ${ }^{22}$

Neste ponto, Bergson é, no espírito como na letra, profundamente biraniano. De facto, é Maine de Biran quem denuncia pela primeira vez a tese do paralelismo nestes termos:

\begin{abstract}
Uma inteligência superior que lesse o cérebro de fora para dentro, e que conhecesse de avanço as relações secretas dos seus movimentos orgânicos com as ideias ou simplesmente o seu paralelismo, descobriria através da representação de uns toda a série das outras, do mesmo modo que penetramos no segredo dos pensamentos de um autor por meio dos caracteres escritos ou impressos que no los representam ${ }^{23}$.
\end{abstract}

Ao comentar esta evidente proximidade de formulações e perspetivas, P. Montebello notou com rigor em que medida é original e percursora a posição de Biran: o filósofo de Bergerac identifica criticamente, "sem dúvida pela primeira vez na história da metafísica, o postulado da legibilidade do espírito" 24 e, de modo consequente, será igualmente o primeiro a denunciar os limites da hipótese metafísica de uma manifestação do moral no físico, do consciente sobre o relevo exterior do anatómico-fisiológico, do pensamento no mapa das localizações neurológicas. Para Biran, é o "dogma implícito e jamais elucidado enquanto tal da psicofisiologia" 25 , segundo o qual deve existir pelo menos uma equivalência (se não uma relação de produção, ou mesmo de identidade) entre o físico e o moral, entre um "estado cerebral" e um "estado mental", que opera tais teses:

Tal é o fundo [argumenta Biran] de uma hipótese que, se se aceita uma vez o seu princípio, se encontrará perfeitamente ligada em todas as suas consequências e parecerá converter ou traduzir realmente a ciência interior das ideias e das faculdades do espírito humano em uma espécie de dinâmica exterior ou de teoria do movimento das fibras do cérebro ${ }^{26}$.

\footnotetext{
${ }^{22}$ Bergson, L'énergie spirituelle, 192.

${ }^{23}$ Maine de Biran, Rapports du physique et du moral de l'homme, in Euvres de Maine de Biran, t. VI, (Paris: Vrin, 1984), 65.

${ }^{24}$ Montebello, Nature et Subjectivité, 65.

${ }^{25}$ Montebello, Nature et Subjectivité, 65.

${ }^{26}$ Maine de Biran, Rapports, 46.
} 
Em tal hipótese, uma questão de fundo é sempre ignorada (nomeadamente por todos quantos, no contexto do estudo do homem, não se protegem suficientemente da fisica ${ }^{27}$ ) quando se aceita cegamente o postulado de uma traduzibilidade do pensamento no cérebro. Poderíamos formulá-la nestes termos: o que poderá legitimar epistemologicamente que se tome por conhecido na terceira pessoa (sob o modelo do olhar e do tocar) o que permanece da ordem da primeira pessoa ou do sentido íntimo? O que poderá justificar que se ignore - como essencial para o conhecimento do propriamente humano do modo de ser humano -a diferença entre o que se pode observar em um $l_{o c a l}{ }^{28}$ (do cérebro, por exemplo) e o que, não tendo imagem $^{29}$ que o traduza no exterior (como o pensamento ou a consciência de si) apenas é evidente ao sentido íntimo?

Estas questões, pressente Biran, começam a ser ignoradas pelo ar do tempo e é cada vez mais difícil não esquecer que, em relação aos detalhes hipotéticos da complexa mecânica cerebral, "se trata apenas de signos sensíveis destinados a representar as operações reflexivas dos fenómenos interiores" ${ }^{30}$, e não esses próprios fenómenos interiores. $\mathrm{O}$ apagamento da referida diferença é, portanto, perigosa já que ilude o problema fundamental de saber se, de um ponto de vista epistemológico, se pode conceber realmente algum tipo de analogia entre o que se conhece como objeto e o que não se pode conhecer senão na respetiva "fonte" ${ }^{31}$ subjetiva. No fundo, é a questão sobre o estatuto do sujeito e da irredutibilidade da própria subjetividade que está aqui em causa: na eventualidade de se alcançar uma descrição completa do mundo e do ser humano seguindo uma verdadeira e estrita teoria física que fosse capaz de estudar todas as partículas, forças e campos que compõem a realidade e, assim, igualmente dar conta de todas as conexões, regiões e elementos neurofisiológicos que formam o humano, oferecendo coordenadas espaciais e explicações observáveis para tudo o que se passa no humano, resistiria ainda a questão mais decisiva para mim: onde e como me encontraria $\mathrm{eu}^{32} \mathrm{em}$ tal mundo? Onde e como, em tal mundo de objetos, se encontraria "o

\footnotetext{
${ }^{27}$ Maine de Biran, Rapports, $5:$ :C'est en ayant égard sans doute à cette contrariété dans la tendance et moyens des deux sciences que Newton, touchant le nœud de la question qui nous occupe, s'écriait ô physique! préservetoi de la métaphysique; c'est aussi en ayant égard à l'observation nécessairement double des deux classes de phénomènes, dont le mélange et la confusion offrent tant d'erreurs et d'illusions et de mécomptes, que nous pouvons nous écrier à notre tour, ô psychologue ! préserve-toi de la physique. 》

${ }^{28}$ Maine de Biran, Rapports, $60 ; 70$ : «c'est dis-je, sur une telle confusion d'idées et de principes que me parait se fonder toute hypothèse imaginée pour localiser (...). »

${ }^{29}$ Maine de Biran, Rapports, 38

${ }^{30}$ Maine de Biran, Rapports, 50

${ }^{31}$ Maine de Biran, Rapports, 47.

${ }^{32} \mathrm{~A}$ atualidade do questionamento biraniano pode ser avaliado, nomeadamente no contexto do debate em redor do problema mente-cérebro, pela sua proximidade, por exemplo, com as formulações de Th. Nagel em textos como The View from Nowhere (Oxford: Oxford University Press, 1986).
} 
sujeito individual do pensamento" 33 , ou seja, onde e como se encontraria algo como a "simplicidade metafísica do $e u$ " existindo ou apercebendo-se $u m$ no ato de pensar ou de se saber $^{34}$ ?

\section{Um "ponto de vista" interior.}

Como é sabido, Maine de Biran enfrenta esta questão do começo do pensar(-se) ou do facto de consciência (pelo qual começo a existir como sujeito) formulando a sua famosa e elegante teoria do esforço primitivo. Para o filósofo de Bergerac, é nas raízes de uma força própria (e sui generis) de vontade e de ação ${ }^{35}$ atualmente aplicada a mover o corpo $^{36}$,que sabemos que a certeza de si (o eu ${ }^{37}$ ) "se identificará completamente com o sentimento primitivo da nossa existência individual na conceção reflexiva de uma força que apenas se torna viva ou consciente dela própria pelo seu desenrolar atual sobre o seu termo de aplicação apropriado" "38 - que é o corpo próprio interiormente resistente. A origem da certeza apercetiva depende, portanto, da relação "interior" dual (mas não dualista) entre dois elementos, distintos mas não separados, que são a força "híper-orgânica" da vontade e o corpo apropriado de uma resistência interiorizada. Uma tal "distinção não separada" dos elementos da dualidade primitiva é fulcral se queremos entender o sentido primitivo do pensamento: tais elementos são, ao mesmo tempo, realmente diferentes e realmente nunca separados, o que implica que não são sequer concebíveis, seja de que modo for, como absolutamente exteriores à própria relação que formam e que não é senão o eu apercetivo. Perseverantes na relação dual primitiva, o corpo da resistência muscular não é o corpo objetivo, mas um corpo próprio sem imagem que se desvenda na força da vontade; a força da vontade, por seu lado, não é uma realidade espiritual, mas uma força própria do indivíduo que se desvenda no corpo apropriado. Ao formarem a unidade dual da relação primitiva, tais elementos não são senão o ato irrepresentável que o eu ativo e incarnado (o eu do pensar-se, do facto de consciência) real e primitivamente é. Neste sentido, a única forma de apreender os elementos do esforço é

\footnotetext{
${ }^{33}$ Maine de Biran, Rapports, 35.

${ }^{34}$ Nagel, The View from Nowhere, 654

${ }^{35}$ Maine de Biran, Mémoire sur la décomposition de la pensée, in Cuvres de Maine de Biran, t. III (Paris :Vrin, 1988), 102.

${ }^{36}$ Maine de Biran, Essai sur les fondements de la psychologie (2 vol.),in Euvres de Maine de Biran, t. VII-1 (Paris : Vrin, 2001), 9.

${ }^{37}$ Maine de Biran,Décomposition, 102. Cf., num mesmo sentido, Maine de Biran, Essai, I, 123: «Le sens interne de l'effort ne peut au contraire être mis en jeu que par cette force intérieure et sui generis que nous appelons volonté avec laquelle s'identifie complètement ce que nous appelons notre moi»

${ }^{38}$ Maine de Biran, Décomposition,125.
} 
fazendo um com a sua distinção não separada; o mesmo é dizer que a evidência do pensamento, do eu apercetivo jamais se poderá descortinar quer do lado de um corpo puramente objetivo e respetivas regularidades materiais (passíveis de serem investigadas sob o modelo da física), quer do lado de um "espírito puramente espiritual" 39 (seja nas suas versões antigas e homunculares, seja nas contemporâneas conceções de uma mente, ou de um self). Apenas na própria relação de esforço - e durante a sua vigência -, apenas sendo essa distinção não separada, esse ato incarnado a certeza de si se constitui: é então uma realidade (um facto, dirá Biran) que eu me sei primitivamente como sujeito e não, justamente, como objeto.

Assim, deve compreender-se que o problema da consciência apercetiva não é um problema científico, cuja solução dependeria da análise dos factos exteriores localizáveis sobre a matéria físico-fisiológica; outrossim, trata-se de um problema filosófico que corresponde à investigação do estado ativo, individualizado, subjetivo em que sabemos que estamos em nós próprio. Ora, um tal tipo de investigação não pode ser alcançado por uma abordagem que julga possível "ver e tocar o pensamento" e que, desse modo, procurará o pensamento (o $e u$, a consciência) onde nunca será possível encontrá-lo: num local, numa imagem; ou seja, fora da relação irrepresentável de esforço com a qual eu faço um no sentimento intimo de mim próprio. A dimensão filosófica do problema em questão implica, portanto, o reconhecimento da evidente disparidade que existe entre o que se convencionou apelidar de "ponto de vista da terceira pessoa - o "ponto de vista exterior", segundo Biran (servido pela faculdade da "imaginação") ${ }^{40}$ - e o ponto de vista da "primeira pessoa"- que Biran identificou como "ponto de vista interior" (o ponto de vista da evidência apercetiva, que se apoia na faculdade de reflexão concentrada) ${ }^{41}$.

$\mathrm{Na}$ verdade, o que parece ser claro a este propósito é que "haverá sempre heterogeneidade absoluta ou falta completa de analogia entre as duas ordens de facto" 42 que se oferecem a cada um dos dois pontos de vista. E tal disparidade, como nota Maine de Biran, não pode ser menosprezada, já que corresponde à diferença irredutível que existe entre a

\footnotetext{
${ }^{39}$ Montebello, Nature et Subjectivité, 104.

${ }^{40}$ Maine de Biran, Discours à la société médicale de Bergerac, in ID,Euvres de Maine de Biran, t. V, (Paris : Vrin, 1984), 50.

${ }^{41}$ Maine de Biran, Discours, 50.

${ }^{42}$ Maine de Biran, Rapports, 48.
} 
simplicidade metafísica do eu existente ou apercebendo-se um num ato de pensamento (...) [e a] espécie de simplicidade física atribuída objetivamente a tal átomo, a tal ponto indivisível considerado como o lugar da alma ${ }^{43}$.

Considere-se o seguinte exemplo para avaliar o alcance da tese biraniana: estou sentado com um cotovelo apoiado no braço da cadeira e, com os dedos da mão transformados numa espécie de apoia-livros, seguro o queixo numa pose que lembra, remota e muito desajeitadamente, o Pensador de Rodin; tenho a testa franzida e o olhar perdido. Imagine-se agora que alguém em frente a mim me observa e que, mais ainda, um aparelho de imagiologia cerebral acompanha ao vivo o funcionamento do meu cérebro tornando-o acessível à observação. Quem me observa naquela pose de alheamento poderá certamente testemunhar que vê uma pessoa "sentada a pensar"; através da máquina de imagiologia confirmar-se-á a atividade cerebral e, ao analisar as colorações que se acendem no écran, poderão alguns peritos afirmar que se ve o pensamento ${ }^{44}$. Na verdade, durante aquele período "eu pensei um pouco", depois "caí num pequeno devaneio" e, finalmente "recordei algo". Sei-o sem ter feito qualquer observação de mim mesmo sobre um espelho, ou qualquer análise direta do meu cérebro. Diria Biran que segui apenas, por um gesto de reflexão concentrada, as dimensões interiores de individuação do esforço apercetivo que atravessam as diferenciações das faculdades enquanto subjetivamente descobertas e significadas. Aqueles observadores exteriores enganar-se-iam, portanto, sobre o que realmente se passaria em mim; e, desse modo, notaria Biran, cairiam na situação bizarra de, tendo eles próprios em si mesmos o acesso direto à certeza apercetiva de si e à diferenciação das faculdades, substituírem essa fonte de evidência por uma análise exterior,sempre infiel por comparação com a evidência interior (no nosso exemplo, eu não teria apenas pensado, mas também sonhado e recordado). Move-os, argumenta Biran, não o respeito pelos factos (na sua diferença), mas uma "metafísica da manifestação" 45 que procura o que é do eu fora ou independentemente do $e u$.

Mas, justamente,

se temos ou podemos ter diretamente a aperceção interna ou o sentimento imediato, independentemente de qualquer conceção imaginária dos jogos ou movimentos das fibras, que necessidade temos de recorrer a esses meios? Quando temos a coisa significada, para que serve o signo? Quando o representado está lá, por quê ir procurar

\footnotetext{
${ }^{43}$ Maine de Biran, Rapports, 66.

${ }^{44}$ Veja-se a afirmação de J.P. Changeux em J.P. Changeux et P. Ricœur, Ce qui nous fait penser. La nature et la règle (Paris : Éditions Odile Jacob, 1998), 69.

${ }^{45}$ Pierre Montebello,La décomposition de la pensée. Dualité et empirisme transcendantal chez Maine de Biran(Grenoble : Million, 1994), 173.
} 
assim tão longe o representante? Se nos é dado comunicar imediatamente com o nosso pensamento, para quê a ajuda de um intermediário exterior que pode ser infiel? ${ }^{46}$

O que fica dito não significa, no entanto, que o plano da "evidência apercetiva" a que se refere Maine de Biran corresponda à descoberta de putativos novos dados, que fossem apenas acessíveis por "mim próprio", dados suplementares em relação à observação físicofisiológica e que "eu" poderia consultar como se estivessem armazenados em um reservatório de verdades inacessíveis. Não são novos dados que se encontram "no interior", mas "factos" distintos daqueles que se oferecem à observação exterior, factos que apenas aparecem a um ponto de vista interior, ou seja, que apenas são o que são porque (e enquanto) eu faço subjetivamente um com eles. Assim, do que aqui se trata, seguindo a perspetiva do filósofo de Bergerac, é de reconhecer a irredutibilidade do estatuto subjetivo do sujeito do pensamento. Mas não só: igualmente há que admitir - eis o decisivo - a anterioridade epistemológica de tal estatuto que, forçosamente, desaparece quando se pretende analisá-lo primeiro a partir do estatuto objetivo das respetivas condições materiais. Quando assim se intenta inverter a ordem do conhecimento, acontece rapidamente substituir-se equivocamente o que se pretenderia de facto estudar (o fenómeno do pensamento enquanto pensamento de si) pelos "signos" orgânicos que se crê serem a respetiva manifestação. Ora, de acordo com Biran, quando "os signos orgânicos" se tornam o objeto principal de estudo ${ }^{47}$, rapidamente se julgarão legitimadas todas as passagens entre o ponto de vista interior e o ponto de vista exterior, entre o que se estuda no cérebro e o que se sabe no estado de conscium ou compos sui; e uma vez assim apagadas as diferenças entre pontos de vista, algo mais se seguirá: sob a pressão do fascínio exercido sobre os "espíritos mais apressados" pelo primado da observação experimental, o "objeto principal de estudo" irá ficando cada vez mais afastado, cada vez mais obliterado pela apaziguadora segurança dos signos ou relevo sensível que se pode ver e tocar - ao ponto de tais signos orgânicos subitamente se tornarem o único objeto de estudo.

Aí chegados, acontecerá enfim reduzir-se o ponto de vista interior ao ponto de vista exterior, ou seja, torna-se aceitável sustentar que uma vez explicado o fisiológico, se esclarece ao mesmo tempo o psicológico, de que cartografando o cérebro se manifesta analogamente o pensamento, a consciência de si, o eu - de que, enfim, haverá uma relação causal de dependência ${ }^{48}$ entre realidades tão diferentes como o pensamento e o cérebro. Neste sentido, será ainda sobre o pano de fundo da tese do paralelismo que Cabanis poderá afirmar - assim

\footnotetext{
${ }^{46}$ Maine de Biran, Rapports, 49

${ }^{47}$ Maine de Biran, Rapports, 49

${ }^{48}$ Maine de Biran, Rapports, 41.
} 
se tornando exemplo paradigmático de um equívoco -, de modo literal e não figurado, que "o cérebro faz a secreção orgânica do pensamento (...) como o estômago faz secreção do suco gástrico (...). $" 49$

\section{Considerações finais.}

Bergson nada dirá de substancialmente diferente de Biran no momento de, pela sua parte, renovar a mesma crítica à ilusão resistente de um cérebro produtor do pensamento. Sobre a base de um acordo de fundo e partilhando o mesmo diagnóstico biraniano sobre a origem filosófica remota - ambos os filósofos fazendo-a remontar à "escola de Descartes" ao "cartesianismo" 51 - da ideologia da manifestação que permite tal suposição de uma relação causal de produção, Bergson afirmará: a principal consequência do paralelismo, a sua "hipótese mais subtil" 52 é a tese segundo a qual o cérebro "poderia criar, ocasionar, ou quando muito exprimir a representação dos objetos" ${ }^{23}$.

Uma vez formulada, no entanto, sustentará Bergson a tese "destrói-se a si mesma" Biran, por seu turno, havia já diagnosticado a que ponto a referida tese, com a qual se procura explicar o conjunto dos "fenómenos do $e u$ através das propriedades da organização (animal)", é efetivamente "vazia". ${ }^{55}$ Em Biran, a crítica à tese do paralelismo e às suas consequências informará a investigação sobre o facto de consciência, sobre a teoria dos pontos de vista e, no limite das possibilidades do ponto de vista interior, operará ainda na possibilidade de investigar o movimento absoluto do existir que se faz, ao mesmo tempo, esforço e consciência, ontogénese individual e ordem das faculdades ${ }^{56}$. Para o leitor competente de Bergson, este fundo biraniano será certamente significativo se se recordar, por exemplo, a reflexão bergsoniana sobre o cérebro como órgão de ação e não de representação ${ }^{57}$, bem como a intuição revolucionária, que acompanha tal análise, de que há menos (e não mais) na representação do que no Todo das imagens, menos na "minha" perceção do que na presença

\footnotetext{
${ }^{49}$ Maine de Biran, Rapports, 37.

${ }^{50}$ Maine de Biran, Rapports, 19-20 ; 92.

${ }^{51}$ Bergson, L'énergie spirituelle, 192.

${ }^{52}$ Bergson, L'énergie spirituelle, 193.

${ }^{53}$ Bergson, L'énergie spirituelle, 203.

${ }^{54}$ Bergson, L'énergie spirituelle, 203.

${ }^{55}$ Maine de Biran, Rapports, 89.

${ }^{56}$ Montebello, Nature et Subjectivité, 106.

57 Ainda que sobre o funcionamento do cérebro Biran e Bergson quase nada poderiam ter sabido de seguro e rigoroso, o reconhecimento de um problema filosófico específico, que hoje continua a chamar a pensar, garantelhes a atualidade.
} 
de um Todo ${ }^{58}$ que dura e encerra as suas próprias condições de desvelamento. Algo de profundamente filosófico se encontra numa tal aproximação que, então, não ilude mas ajuda a perceber os caminhos dissemelhantes destes dois projetos maiores.

\section{REFERÊNCIAS BIBLIOGRÁFICAS}

BARBARAS, Renaud. Introduction à une philosophie de la vie. Paris: Vrin, 2008.

BERGSON, Henri. "L'âme et le corps", in ID L'énergie spirituelle (édition critique sous la direction de F. Worms). Paris: PUF, Quadrige, 2009.

BERGSON, Henri. "La Philosophie Française", in Revue de Paris, mai-1915, cit. in H. Gouhier, "Maine de Biran et Bergson », in Les études bergsoniennes, vol. 1. Paris: Albin Michel, 1948.

BERGSON, Henri. "Le cerveau et la pensée, une illusion philosophique", in ID L'énergie spirituelle (édition critique sous la direction de F. Worms). Paris: PUF, Quadrige, 2009.

BERGSON, Henri. « Rapports sur le prix Bordin. Concours ayant pour sujet Maine de Biran et sa place dans la philosophie moderne », in ID, Mélanges, Édition du Centenaire , Paris: PUF, 1972.

CHANGEUX et RICEUR. Ce qui nous fait penser. La nature et la règle. Paris: Éditions Odile Jacob, 1998.

DELEUZE, Gilles. «L'Immanence: une vie », in Philosophie, 47, 1995.

GOUHIER, Henri. «Maine de Biran et Bergson», in Les études bergsoniennes, vol. 1. Paris: Albin Michel, 1948.

LE ROY, George. L'expérience de l'effort et de la grâce chez Maine de Biran. Paris: Boivin \& Cie., 1937.

MADINIER, Gabriel. Conscience et mouvement. Étude sur la philosophie française de Condillac à Bergson. Paris: Alcan, 1938.

MAINE DE BIRAN. Discours à la société médicale de Bergerac, in ID, Euvres de Maine de Biran, t. V. Paris: Vrin, 1984.

MAINE DE BIRAN. Essai sur les fondements de la psychologie (2 vol.), in CEuvres de Maine de Biran, t. VII-1. Paris: Vrin, 2001.

MAINE DE BIRAN. Mémoire sur la décomposition de la pensée, in Euvres de Maine de Biran, t. III, Paris:Vrin, 1988.

MAINE DE BIRAN. Rapports du physique et du moral de l'homme, in Euvres de Maine de Biran, t. VI. Paris: Vrin, 1984.

MONTEBELLO, Pierre. La décomposition de la pensée. Dualité et empirisme transcendantal chez Maine de Biran. Grenoble: Million, 1994.

MONTEBELLO, Pierre. Nature et Subjectivité. Grenoble: Millon, 2007.

NAGEL, Th. The View from Nowhere. Oxford: Oxford University Press, 1986.

THIBAUD, Marguerite. L'effort chez Maine de Biran et Bergson. Grenoble: Imprimerie Allier \& Fils, 1939.

${ }^{58}$ Montebello, Nature et Subjectivité, 106. 